\title{
An alternative approach for the mainframe of Flabby Ridge
}

\author{
Varun Kumar $^{1 *}$, Jyotsna Seth ${ }^{2}$, Rahul Dhami ${ }^{3}$, Megha Sagar \\ ${ }^{1}$ Professor, ${ }^{2,3}$ Reader, ${ }^{4}$ PG IInd Year, Dept. of Prosthodontics, Seema Dental College \& Hospital, Rishikesh, Uttarakhand, India
}

*Corresponding Author: Varun Kumar

Email: drvarun_smile@yahoo.co.in

\begin{abstract}
A fibrous or flabby ridge is a superficial area of mobile soft tissue affecting maxillary or mandibular alveolar ridges. It develops when a hyperplastic tissue replaces the bone, often seen in long-term denture wearers and clearly related to residual alveolar ridge resorption. The prevalence studies have shown that it most commonly affects the upper anterior region in denture wearers and affects edentulous maxilla $(24 \%)$ and edentulous mandible $(5 \%)$ when alveolar bone is replaced by hypertrophic soft tissues. ${ }^{1}$ The displacement of this mobile tissue occurs easily during impression making causing distortion and also during mastication resulting in loss of peripheral seal. ${ }^{2}$ Although these movable tissues does not provide good retention but should be recorded to ensure good support. ${ }^{3,4}$ Also, when the new denture is adjusted into the mouth, patient's complains of pain caused by compression of tissues between the denture and the bone. Therefore, the intaglio surface of the dentures or the surface from where pressure is transmitted should have maximum possible area to reduce pressure on the oral mucosa. During impression making this mobile tissue should be recorded without distortion using special techniques that would provide for adequate retention, stability and support in a complete denture. ${ }^{5}$ Flabby ridges have also been reported in cases with maxillary complete dentures opposing mandibular natural teeth. In 1972 Kelly described the term " combination syndrome to the changes in patients wearing maxillary complete dentures opposing mandibular anterior teeth. ${ }^{6}$ The case presented here describes a special technique for such tissues for successful esthetic, functional and phonetic rehabilitation of an edentulous patient with maxillary anterior flabby tissue since conventional mucocompressive and muco-static impression techniques are not suitable for such cases, as the displaceable soft tissue exhibits 'recoil' upon compression. $^{7}$
\end{abstract}

Keywords: Flabby tissue, Window technique, Combination syndrome.

\section{Case Report}

A 66 -year- old female patient reported in the Department of Oral and Maxillofacial Prosthodontics and Oral Implantology with a chief complaint of ill-fitting removable partial denture and difficulty in chewing food. The patient has been a denture wearer for past 8 years and she has been reported in the department for the third replacement for the denture. On intra-oral examination a completely edentulous maxillary arch was observed with flabby tissue in the anterior region opposing the mandibular natural anterior teeth without proper posterior occlusal support. When pressurized with the blunt end of mouth mirror blanching of flabby tissue was noted. Various treatment modalities were given to the patient like preprosthetic surgical excision of flabby tissue, implantsupported dentures, etc. But the patient denied for the options explained to her and was more interested for the conventional approach of denture construction. Therefore, other options were ruled out and the final treatment plan was executed including the use of window impression technique for the maxillary arch.

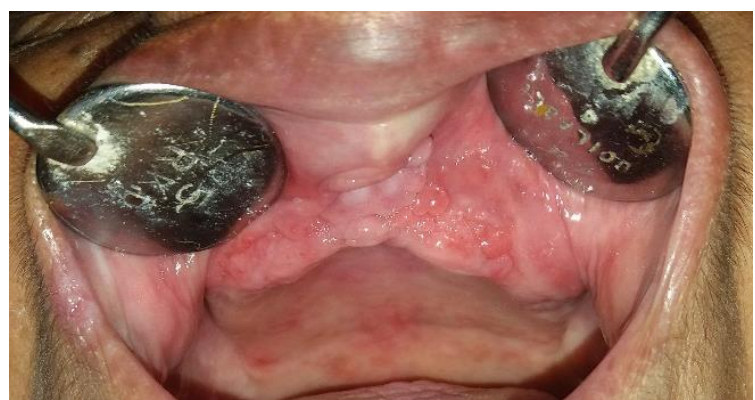

Fig. 1: Flabby Tissue in the anterior maxilla

\section{Technique}

The primary impression of the maxillary denture bearing area was made using a mucostatic impression material (Alginate: Dentsply), to ensure minimal distortion of the displaceable flabby tissue. (Fig. 1) The cast was then poured using type II gypsum product (Dental plaster). A custom tray was fabricated over this cast so as to make the final impressions. An indelible pencil was used to mark the identified flabby areas on the cast. A closely fitting custom tray was fabricated in the conventional manner using auto-polymerizing acrylic resin with additional thickness of the modelling wax over the moveable areas identified on the cast apart from the wax spacer adapted over the non-displaceable areas. (Fig. 2) The tray was then checked in the patients mouth for any overextension. The border moulding was carried out in segments using type I low fusing impression compound (Green stick compound) conventionally in the maxillary arch until the functional depth of the sulcus was recorded. (Fig. 3). A window was created in the maxillary anterior region corresponding to the flabby tissues. (Fig. 4) Then the wax spacer was removed from the custom tray and the impression was made using the medium body elastomeric material. The tray was then removed from the mouth and the impression material in the region of the flabby tissue was removed using a Bard-parker knife. After providing relief the custom tray was loaded in this region with light-body elastomeric impression material.( Fig. 5) Beading and boxing was carried out for the final impression and the master cast was poured using type III gypsum product (Dental stone). The denture was fabricated in the conventional manner which had good retention and support. (Fig. 6) 


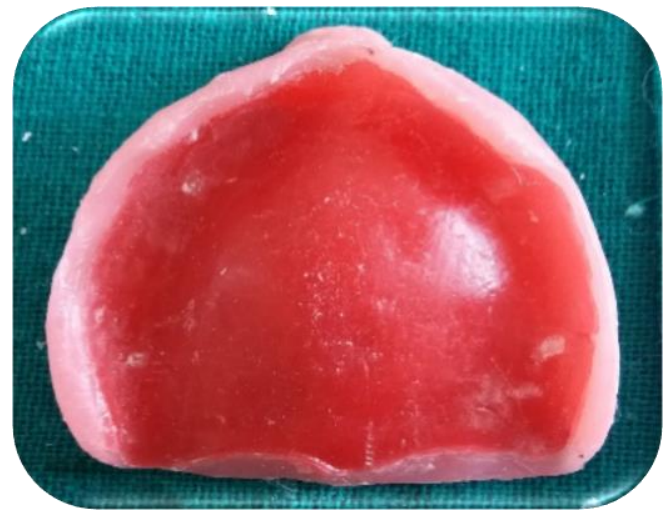

Fig. 2: Custom tray

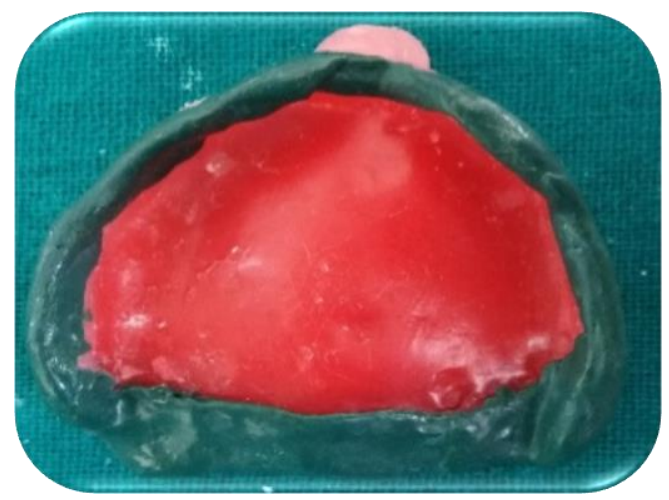

Fig. 3: Border moulding

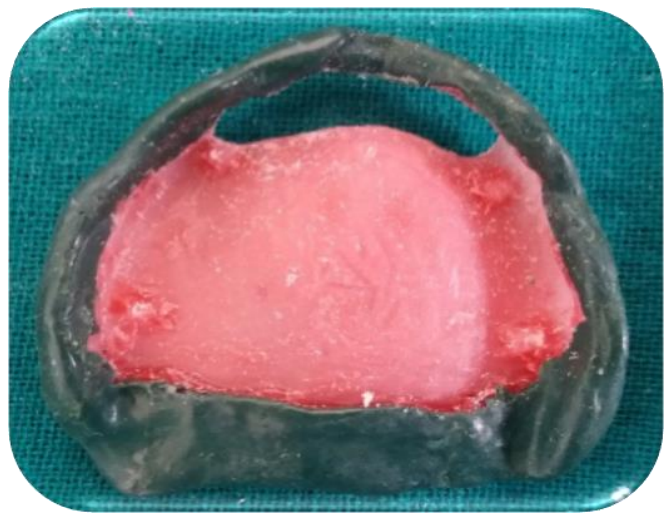

Fig. 4: Modified custom tray to accommodate flabby tissue

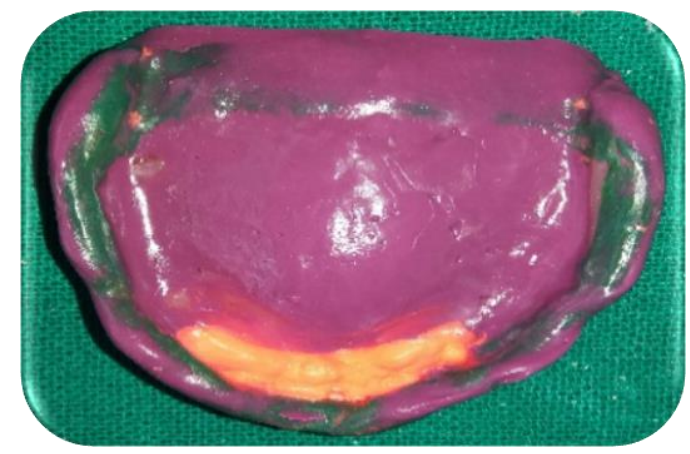

Fig. 5: Final impression

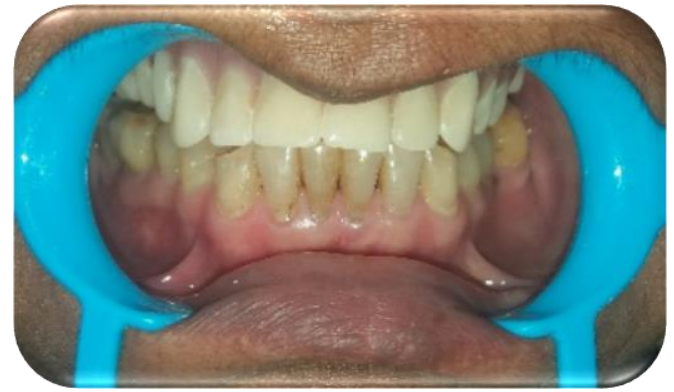

Fig. 6: Post-op

\section{Discussion}

With proper prosthodontic approach, flabby ridge can be successfully treated either alone or in interdisciplinary combination with surgery. Surgical removal of flabby tissue is possible if there is adequate bone height. However, it results in short sulcus depth that further needs a small surgical intervention i.e. vestibuloplasty. Conventional impression techniques used to record such flabby tissues often results in unretentive and unstable dentures. Creating holes/ windows or wax reliefs decreases the hydraulic pressure while impressing flabby areas, thus minimizing the distortion/ displacement of hypermobile tissues. Utilizing these alternatives while making secondary impression can be useful in recording flabby tissues in their anatomic or undistorted form. ${ }^{6,8}$

Liddlelow in 1964 described a technique whereby two separate impression materials were used in a custom tray (using 'plaster of Paris' over the flabby tissues and zinc oxide eugenol over the normal tissues). In 1964, Osborne described a technique where two separate impression trays and materials were used to separately record the 'flabby' and 'normal' tissues and then related intra-orally. Watt and McGregor in 1986 described a technique where impression compound was applied to a modified custom tray and a wash impression with zinc-oxide and eugenol was made. ${ }^{9}$

Disadvantages of impression plaster included the material properties that were affected by operator handling technique, Taste and roughness of the material causing the patient to vomit, messiness and a variable setting time due to temperature and humidity. Eugenol is irritating to soft tissues. This material is non- elastic and may fracture if undercuts are present. This led to the search of a better impression material that is compatible with the technique. Advantages of addition silicone impression material such as its shorter setting time, easy to mix, adequate tear strength, extremely high accuracy, absence of any distortion on removal and good dimensional stability suggests that addition silicone can potentially replace the impression plaster in the conventional technique. ${ }^{10}$

\section{Conclusion}

Prosthodontic rehabilitation of a patient with flabby ridge poses a challenge to the dentist. Use of conventional mucostatic, mucocompressive or selective pressure techniques may not be the best method to record such mobile 
tissues and thus modification in these techniques is important. $^{8}$

The suggested method eliminates the excessive displacement of the soft tissues at the secondary impression using a better patient accepted material. Thus, a physiologic and anatomic registration of the attached and the unattached tissue of the denture bearing areas is attained.

\section{Source of funding}

None.

\section{Conflict of interest}

None.

\section{References}

1. Xie Q, Nähri TO, Nevalainen JM. Oral status and prosthetic factors related to residual ridge resorption in elderly subjects. Int J Prosthodont 1997;55:306-13.

2. Crawford RWI, Walmsley AD. A review of prosthodontics management of fibrous ridges. Br Dent J 2005;19;9:715-9.

3. The British Society for the Study of Prosthetic Dentistry. Guidelines in prosthetic and implant dentistry. London: Quintessence, 1996.
4. Carlsson GE. Clinical morbidity and sequelae of treatment with complete dentures. J Prosthet Dent 1998;79:17-23.

5. Lynch CD, Allen PF. Management of the flabby ridge: using contemporary materials to solve an old problem. Br Dent $J$ 2006;200:258-1.

6. Kelly E. Changes caused by a mandibular removable partial denture opposing a maxillary complete denture. J Prosthet Dent 2003;90(3):213-9.

7. Davis D. Complete Dentures: From Planning to Problem Solving. Br Dent J 2004;196:786.

8. Allen F. Management of the flabby ridge in complete denture construction. Dent Update 2005;32:524-8.

9. Lynch CD, Allen PF. Management of the flabby ridge: using contemporary materials to solve an old problem. Br Dent $J$ 2006;200:258e-61.

10. Anusavice KJ, Phillip's Science of Dental Materials, Eleventh Edition, Elsevier, USA, 2007.

How to cite this article: Kumar V, Seth J, Dhami R, Sagar M. An alternative approach for the mainframe of Flabby Ridge. Int J Oral Health Dent 2019;5(3):154-6. 